\title{
Durable clinical benefit from pyrotinib combined with carboplatin in HER2-positive relapsed breast cancer previously treated with taxanes, anthracyclines, and trastuzumab
}

\author{
Wenzhen Zhu, Jianchao Wu, Min Cui, Lin Zhang \\ Zhuhai People's Hospital (Zhuhai Hospital Affiliated with Jinan University), Zhuhai, China \\ Correspondence to: Lin Zhang. Zhuhai People's Hospital (Zhuhai Hospital Affiliated with Jinan University), No. 79 of Kangning Road, Xiangzhou \\ District, Zhuhai 519000, China. Email: xiaolin985985@21cn.com.
}

\begin{abstract}
Patients with human epidermal growth factor receptor 2 (HER2)-positive breast cancer inevitably progressed after a short response to initial trastuzumab treatment, suggesting a possibility of acquiredresistance to trastuzumab. Pyrotinib, an irreversible pan-ErbB receptor tyrosine kinase inhibitor (TKI), has been reported as an effective and safe drug for the treatment of HER2-positive relapsed or metastatic breast cancer. Pyrotinib combined with capecitabine is widely used to treat HER2-positive metastatic breast cancer in patients who have been previously treated with anthracyclines, taxanes, and trastuzumab. However, the efficacy of pyrotinib combined with other chemotherapy drugs is still unclear. Here we report pyrotinib combined with carboplatin in treating a patient with HER2-positive relapsed breast cancer who had acquired resistance to trastuzumab. The patient received three cycles of treatments of pyrotinib (400 $\mathrm{mg}$, orally once per day, days 1-21) combined with carboplatin (600 mg, iv drip, day 1, cycled every 21 days). The patient showed an excellent response to the therapy, including faded rashes on the skin of her breast, no obvious signs of recurrence from the breast magnetic resonance imaging (MRI), decreased skin thickness and cord shadow of the right breast, unchanged degree of right pleural effusion, and no enlarged LN. The patient had a stable disease time of more than four months. Our case provides evidence for the feasibility and efficacy of pyrotinib with carboplatin in treating patients with HER2-positive relapsed or metastatic breast cancer who may develop resistance to trastuzumab.
\end{abstract}

Keywords: HER2 positive; relapsed breast cancer; pyrotinib; carboplatin; case report

Submitted Jun 05, 2020. Accepted for publication Sep 16, 2020.

doi: 10.21037/apm-20-1363

View this article at: http://dx.doi.org/10.21037/apm-20-1363

\section{Introduction}

Pyrotinib is an irreversible receptor tyrosine kinase inhibitor (TKI) used to inhibit multiple ErbB receptors such as human epidermal growth factor receptor 1 (HER1), HER2, and HER4 (1). Pyrotinib has been demonstrated to be an effective and safe drug to treat HER2-positive relapsed and metastatic breast cancers (2-4). For example, $\mathrm{Ma}$ and co-workers reported response to and tolerable adverse effects of pyrotinib therapy in half of 38 patients with heavily pretreated metastatic breast cancer, and determined a maximum tolerated pytotinib dose of $400 \mathrm{mg}$ per day (4). Pyrotinib combined with capecitabine could significantly improve patients' objective response rate (ORR) and median progression-free survival (PFS) time (3), and therefore has been approved for treating HER2-positive advanced or metastatic breast cancer in patients previously treated with taxanes, anthracyclines, and trastuzumab (5). However, the effect of pyrotinib therapy on patients with resistance to trastuzumab has not been fully demonstrated. A phase II trial study showed promising effects of pyrotinib plus capecitabine therapy on patients with HER2-positive metastatic breast cancer previously treated with taxanes, anthracyclines and trastuzumab (3). Specifically, pyrotinib plus capecitabine therapy showed a 
significantly higher objective response rate (79\% vs. 57\%) and median progression free survival (PFS) time (18.1 vs. 7.0 months) compared to lapatinib plus capecitabine therapy. Nevertheless, the treatment effect of pyrotinib combined with other chemotherapy drugs remains unclear, limiting a clinician's selection of chemotherapy drugs.

As a widely used adjuvant chemotherapy drug, carboplatin has been found to own highly synergistic activity with trastuzumab (6-8). One previous study has found that trastuzumab, combined with carboplatin, could significantly improve the clinical outcomes of patients with HER2-positive metastatic breast cancer (9). Therefore, investigating the treatment efficacy of pyrotinib combined with carboplatin could supply significant insights into new therapeutic options for the pyrotinib-based treatment regimen. Here we present a case showing continuous clinical benefits from pyrotinib combined with carboplatin in one patient with HER2-positive relapsed breast cancer previously treated with taxanes, anthracyclines, and trastuzumab. We present the following article in accordance with the CARE reporting checklist (available at http:// dx.doi.org/10.21037/apm-20-1363).

\section{Case history}

A 45-year-old female patient was recruited for the case study. This study was approved by the ethics committee of the host institute. In May 2017, the patient was diagnosed as left breast cancer (Figure 1), invasive ductal carcinoma (IDC), grade III, with lymphatic vessels infiltrated with tumor cells, estrogen receptor (ER) negative $(<1 \%)$, progesterone receptor (PR) negative $(<1 \%)$, HER2 positive (3+ by immunohistochemistry, IHC), Ki67 50\%+, and fluorescence in situ hybridization (FISH) positive (Figure $1 A, B, C, D)$. Then, she underwent a mastectomy of the left breast and left axillary LNs dissection, with 21 of 23 axillary LNs found as metastasized. From May to November 2017, she received adjuvant chemotherapy with four cycles of EC (epirubici, $85 \mathrm{mg} / \mathrm{m}^{2}$, cyclophosphamide $600 \mathrm{mg} / \mathrm{m}^{2}$, iv drip, cycled every 21 days), followed by TH (docetaxel $80 \mathrm{mg} / \mathrm{m}^{2}$, iv drip, cycled every 21 days for 4 cycles; trastuzumab, $8 \mathrm{mg} / \mathrm{kg}$ as the first dosage, $6 \mathrm{mg} / \mathrm{kg}$ in the following cycles, cycled every 21 days for one year). With postoperative radiotherapy and one-year trastuzumab treatment, no recurrence or metastasis was detected in her regular follow-ups until November 2018.

Magnetic resonance imaging (MRI) (Figure 2) on December 26, 2018, showed skin thickening and subcutaneous multiple strip shadows of the right breast, enlarged right axillary LNs, and right pleural effusion, suggesting that tumor infiltration could not be excluded (Figure 2A,B,C). Three months after trastuzumab adjuvant use, she occasionally noticed rashes on the skin of her right breast and left chest wall (Figure 3). However, the patient refused to receive further biopsies because she claimed not to have discomfort.

Three months later, the patient came to seek further treatment because more rashes had occurred on the right breast and left chest wall (Figure 3A). On February 11, 2019, we performed a lumpectomy on her right breast mass with the last MRI result. She was pathologically diagnosed as IDC, grade II, ER-negative, PR negative, HER2 positive, and Ki67 40\%+ (Figure 1E,F,G,H), with tumor cells dispersing in a $5 \times 4.5 \mathrm{~cm}$ area and infiltrating lymphatic vessels in the skin. Meanwhile, biopsies of skin rashes on the right breast and left chest wall were performed, which were identified as IDC, with tumor cells in the skin dermis and lymphatic vessels, ER-negative, PR negative, HER2 positive and Ki67 20\%+. Therefore, the patient was diagnosed with HER2-positive relapsed breast cancer, metastasis from the left to the right breast and chest wall skin. According to the thoracic surgeon's opinion, a pleural puncture biopsy could not be administered due to insufficient pleural effusion.

From February 28, 2019, the patient received treatment of pyrotinib (400 mg, orally once per day, days 1-21) combined with carboplatin $(600 \mathrm{mg}$, iv drip, day1, cycled every 21 days) as the first-line treatment regimen. Four days later, the color of the rashes on the skin faded (Figure 3B). She completed another two cycles of treatments as planned without clear side effects, and then received breast MRI scanning. Compared with the MRI result of December 26, 2018, the skin thickness and cord shadow of the right breast were observed to have decreased (Figure 2D,E,F), while the right pleural effusion remained similar. Moreover, the rash area on the right breast skin had significantly shrunk, and the color of the rashes had faded. No rashes were found on the left chest wall skin (Figure 3C).

The patient did not receive later treatment in our hospital due to personal financial issues. No new rashes were found on the skin of her right breast or her left chest wall after three months (Figure 3D). No obvious signs of recurrence were shown from the breast MRI (Figure 2G,H,I). Also, skin thickness and cord shadow of the right breast was observed to have decreased, while the degree of right pleural effusion remained similar. Finally, no enlarged LN was found. All procedures performed in 

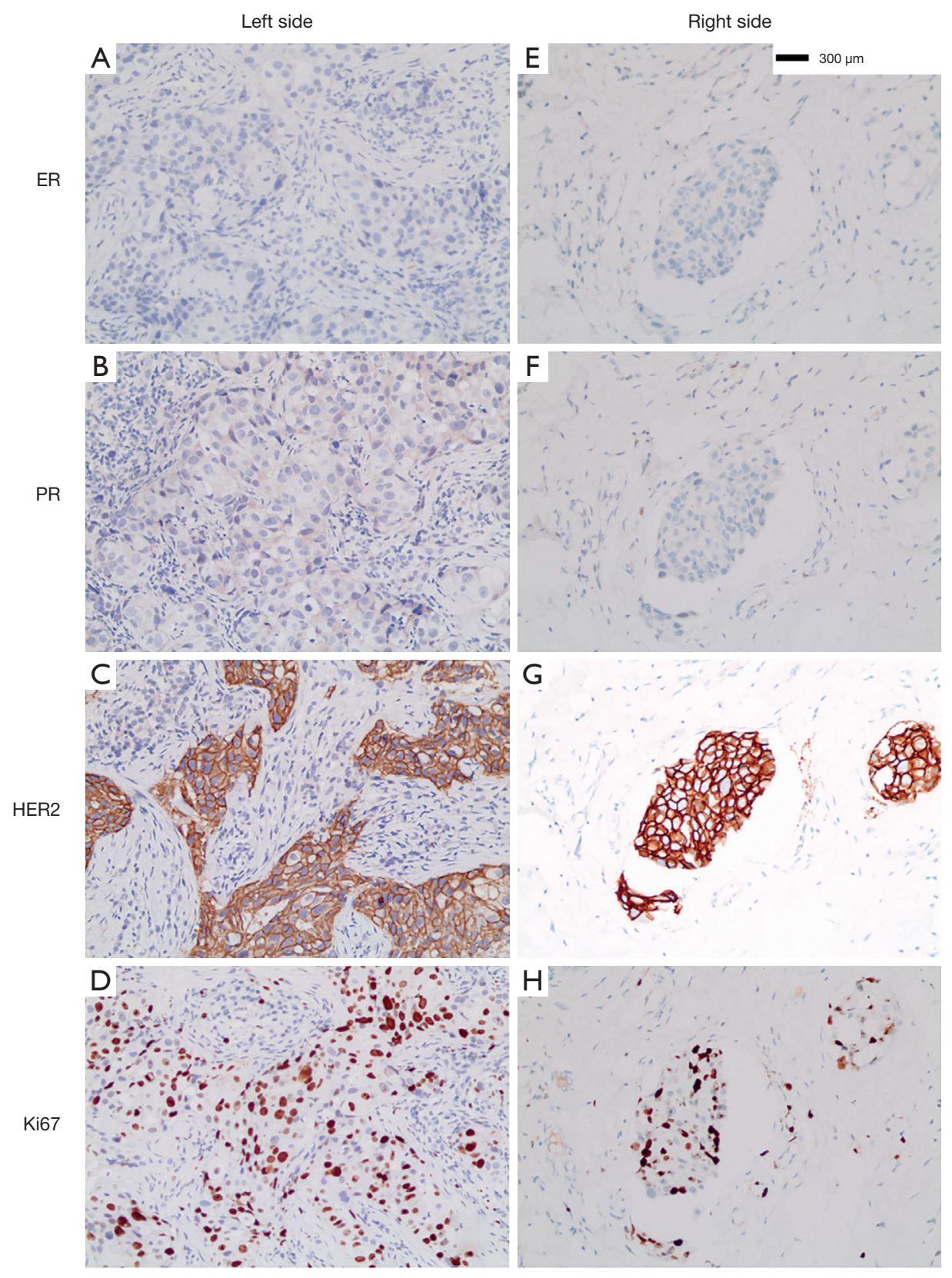

Figure 1 Immunohistochemistry (IHC) analysis of the bilateral breast tumors. (A,B,C,D) The left breast tumor: estrogen receptor (ER) $(<1 \%)$, progesterone receptor $(\mathrm{PR})(<1 \%)$, human epidermal growth factor receptor 2 (HER2) (3+), Ki67 (50\%+). (E,F,G,H) The right breast tumor: ER (<1\%), PR (<1\%), HER2 (3+), Ki67 (40\%+).

studies involving human participants were in accordance with the ethical standards of the institutional and/or national research committee(s) and with the Helsinki Declaration (as revised in 2013). Written informed consent was obtained from the patient.

\section{Discussion}

The patient was diagnosed as breast cancer recurrence three months after one-year trastuzumab adjuvant use, showing possible acquiring of trastuzumab resistance. These findings served as the rationale to change to other HER2 target 

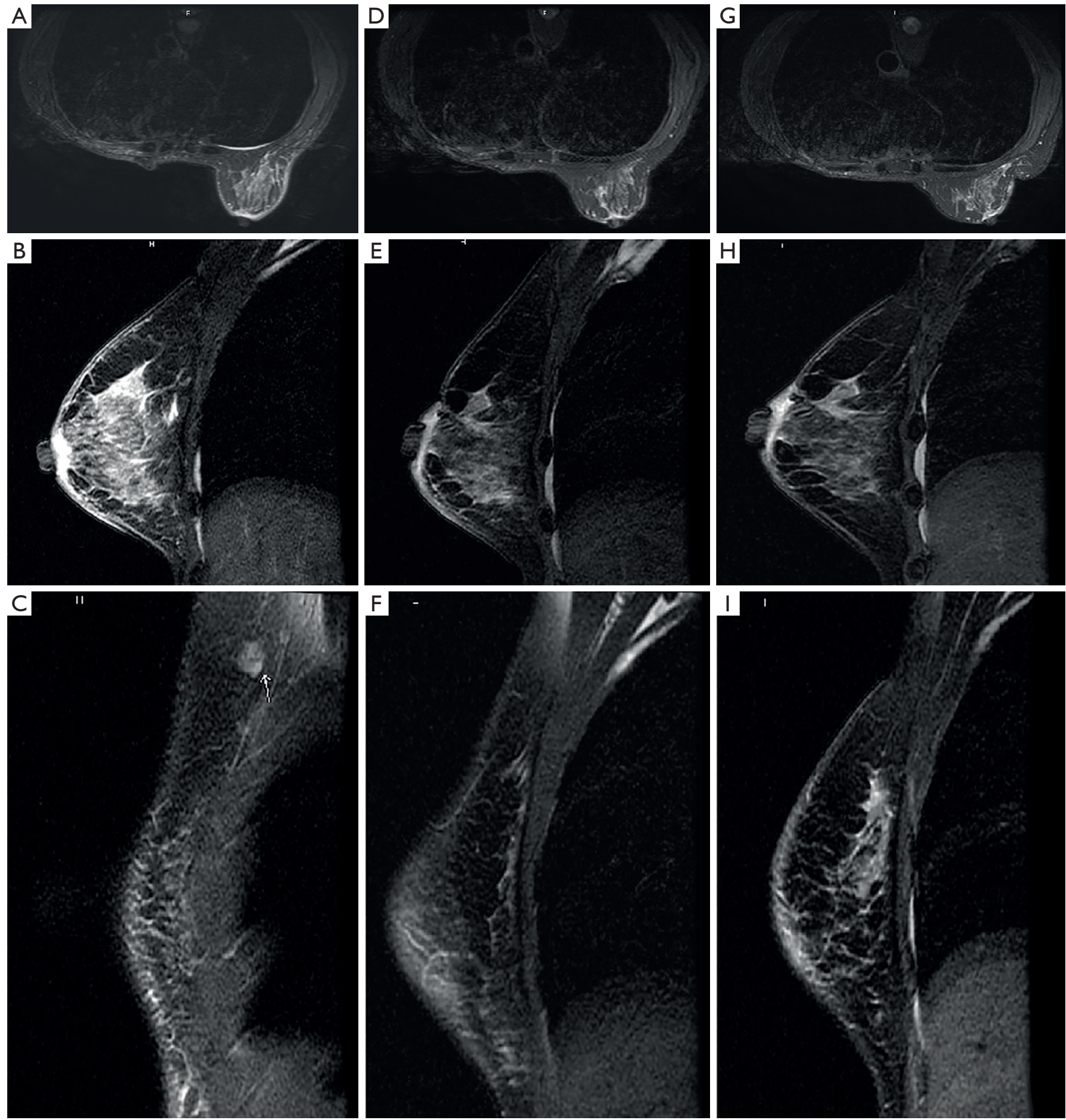

Figure 2 Breast magnetic resonance imaging (MRI). (A,B,C) MRI on December 26, 2018: transverse plane, sagittal plane, enlarged LN of right axilla. (D,E,F) MRI on April 30, 2019: transverse plane, sagittal plane, no enlarged LN of right axilla. (G,H,I) MRI on August 1, 2019: transverse plane, sagittal plane, no enlarged LN of right axilla. MRI, magnetic resonance imaging. 


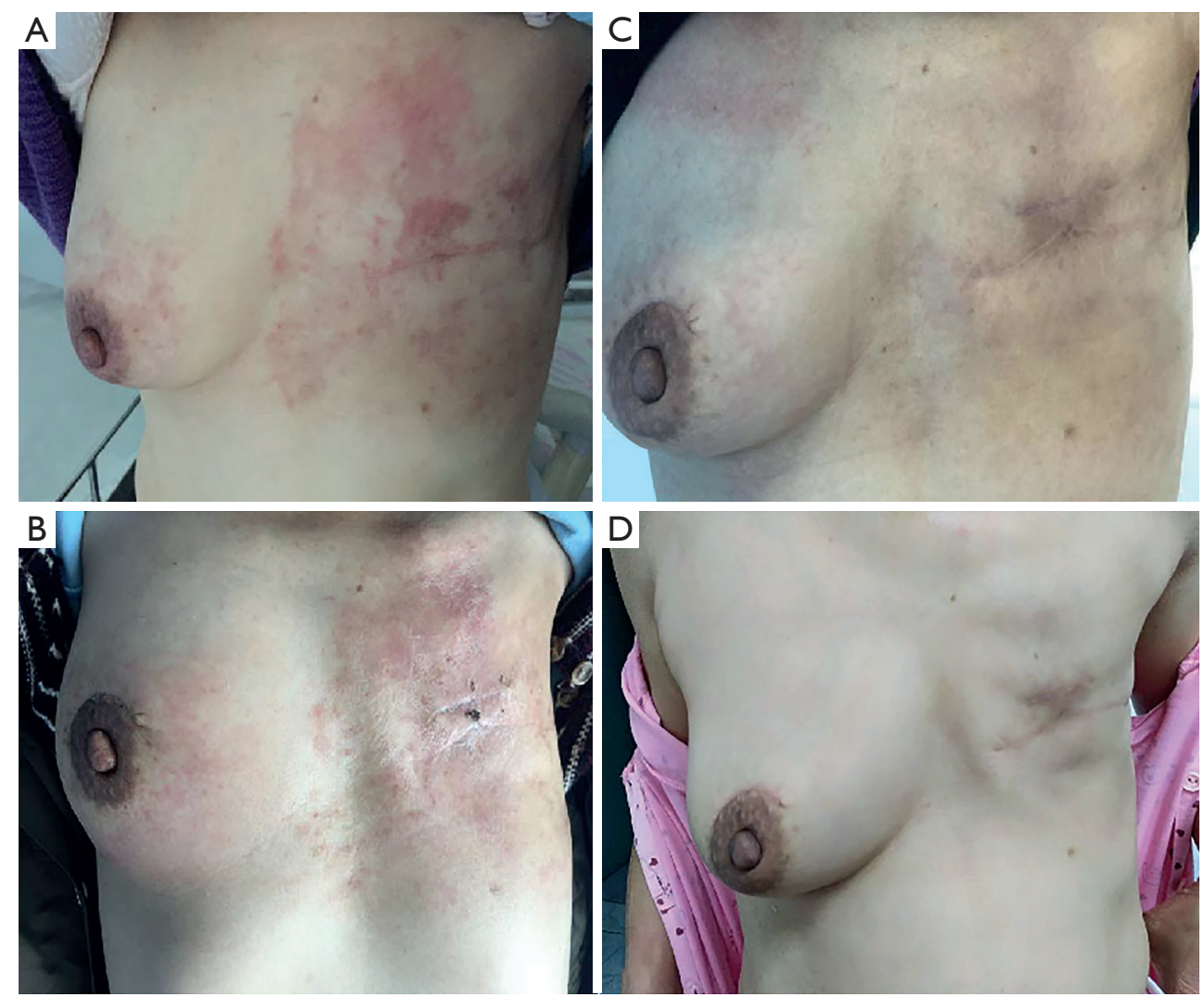

Figure 3 Photos of rashes on the right breast and the left chest skin. (A) The photo was taken before treatment (February 1, 2019): red rashes on the right breast and the left chest wall skin. (B) The picture was taken on the 4th day during the 1st cycle of treatment (March 4, 2019): rashes on the skin faded in color. (C) The picture was taken after 3 cycles of treatment (April 30, 2019): rash shrank and faded in color on the right breast skin. No rashes on the left chest wall skin. (D) The picture was taken 3 months after discontinuing treatment (August 1 , 2019): no rashes on the right breast and the left chest wall skin.

agents. Ma and co-workers observed a durable response to pyrotinib treatment in six patients with the trastuzumabresistant disease. Five patients achieved partial responses, with the most prolonged response duration of 382 days. One trastuzumab-resistant patient with stable disease had a time to progression (TTP) of 128 days. In our case, we implemented three cycles of pyrotinib combined with carboplatin as the later therapy to treat the patient. The patient was evaluated as a stable disease for more than 150 days (from February 28, 2019, to August 1, 2019), which might have been even longer. These results show that the efficacy of pyrotinib combined with carboplatin on our patient is close to pyrotinib combined with capecitabine in other studies. Diarrhea is the most common side effect associated with TKI targeting epidermal growth factor receptor (EGFR)/HER2 (10). The patient in our case took $400 \mathrm{mg}$ of pyrotinib per day and did not exhibit obvious diarrhea during the whole treatment. Also, carboplatin is much cheaper than capecitabine, and therefore may be more affordable for patients in economically underdeveloped regions. In conclusion, our study provides evidence of the feasibility of pyrotinib combined with carboplatin in treating patients who have HER2-positive replaces or metastatic breast cancer previously treated with taxanes, anthracyclines, and trastuzumab.

\section{Acknowledgments}

Funding: None.

\section{Footnote}

Reporting Checklist: The authors have completed the CARE reporting checklist. Available at http://dx.doi.org/10.21037/ 
apm-20-1363

Conflicts of Interest: All authors have completed the ICMJE uniform disclosure form (available at http://dx.doi. org/10.21037/apm-20-1363). The authors have no conflicts of interest to declare.

Ethical Statement: The authors are accountable for all aspects of the work in ensuring that questions related to the accuracy or integrity of any part of the work are appropriately investigated and resolved. All procedures performed in studies involving human participants were in accordance with the ethical standards of the institutional and/or national research committee(s) and with the Helsinki Declaration (as revised in 2013). Written informed consent was obtained from the patient for publication of this study and any accompanying images.

Open Access Statement: This is an Open Access article distributed in accordance with the Creative Commons Attribution-NonCommercial-NoDerivs 4.0 International License (CC BY-NC-ND 4.0), which permits the noncommercial replication and distribution of the article with the strict proviso that no changes or edits are made and the original work is properly cited (including links to both the formal publication through the relevant DOI and the license). See: https://creativecommons.org/licenses/by-nc-nd/4.0/.

\section{References}

1. Zhu Y, Li L, Zhang G, et al. Metabolic characterization of pyrotinib in humans by ultra-performance liquid chromatography/quadrupole time-of-flight mass spectrometry. J Chromatogr B Analyt Technol Biomed Life Sci 2016;1033-1034:117-27.

2. Ma F, Ouyang Q, Li W, et al. Pyrotinib or Lapatinib

Cite this article as: Zhu W, Wu J, Cui M, Zhang L. Durable clinical benefit from pyrotinib combined with carboplatin in HER2-positive relapsed breast cancer previously treated with taxanes, anthracyclines, and trastuzumab: a case report. Ann Palliat Med 2020;9(5):3684-3689. doi: 10.21037/apm-20-1363
Combined With Capecitabine in HER2-Positive Metastatic Breast Cancer With Prior Taxanes, Anthracyclines, and/or Trastuzumab: A Randomized, Phase II Study. J Clin Oncol 2019;37:2610-9.

3. Xu BH, Ma F, Ouyang QC, et al. A randomized phase II trial of pyrotinib plus capecitabine versus lapatinib plus capecitabine in patients with HER2positive metastatic breast cancer previously treated with taxanes, anthracyclines and/or trastuzumab. Cancer Res 2018;78:Abstract PD3-08.

4. Ma F, Li Q, Chen S, et al. Phase I Study and Biomarker Analysis of Pyrotinib, a Novel Irreversible Pan-ErbB Receptor Tyrosine Kinase Inhibitor, in Patients With Human Epidermal Growth Factor Receptor 2-Positive Metastatic Breast Cancer. J Clin Oncol 2017;35:3105-12.

5. Blair HA. Pyrotinib: First Global Approval. Drugs 2018;78:1751-5.

6. Pegram MD, Finn RS, Arzoo K, et al. The effect of HER2/neu overexpression on chemotherapeutic drug sensitivity in human breast and ovarian cancer cells. Oncogene 1997;15:537-47.

7. Pegram M, Hsu S, Lewis G, et al. Inhibitory effects of combinations of HER-2/neu antibody and chemotherapeutic agents used for treatment of human breast cancers. Oncogene 1999;18:2241-51.

8. Pegram MD, Lopez A, Konecny G, et al. Trastuzumab and chemotherapeutics: Drug interactions and synergies. Semin Oncol 2000;27:21-5.

9. Robert N, Leyland-Jones B, Asmar L, et al. Randomized phase III study of trastuzumab, paclitaxel, and carboplatin compared with trastuzumab and paclitaxel in women with HER-2-overexpressing metastatic breast cancer. J Clin Oncol 2006;24:2786-92.

10. Chan A. Neratinib in HER-2-positive breast cancer: results to date and clinical usefulness. Ther Adv Med Oncol 2016;8:339-50. 